\title{
Explaining the global distribution of peak-spectrum variability of sea surface height
}

\author{
Xiaopei Lin, ${ }^{1,2}$ Jiayan Yang, ${ }^{2}$ Dexing $\mathrm{Wu},{ }^{1}$ and Ping Zhai ${ }^{1}$ \\ Received 14 April 2008; revised 27 May 2008; accepted 11 June 2008; published 19 July 2008.
}

[1] A 14-year satellite observation of sea surface height (SSH) reveals an interesting pattern. Along any latitude, there is a frequency at which the $\mathrm{SSH}$ power spectrum peaks, regardless of which hemisphere or oceanic basin. This peak-spectrum frequency is nearly identical to the critical frequency at which the zonal energy propagation of Rossby waves becomes stagnant. The interior ocean adjusts to atmospheric forcing by radiating energy away through Rossby waves. There are two distinct groups of Rossby waves, long ones carry the energy to the west while short ones send the energy to the east. At the critical frequency, these two waves merge and their zonal energy propagation becomes stagnant. Consequently, the energy from atmospheric forcing may accumulate in the ocean interior, and thus result in a spectrum peak. Citation: Lin, X., J. Yang, D. Wu, and P. Zhai (2008), Explaining the global distribution of peak-spectrum variability of sea surface height, Geophys. Res. Lett., 35, L14602, doi:10.1029/2008GL034312.

\section{Introduction}

[2] The ocean and the atmosphere are the two most important components of the earth's climate system. They interact constantly with each other and give rise to changes in the climate system. These two vast bodies of fluids, however, adjust well to such changes and remain largely in equilibrium. Planetary waves, known as Rossby waves in meteorology and oceanography, play the central role in atmosphere-ocean adjustments [Longuet-Higgins, 1964, 1965; Anderson and Gill, 1975]. Oceanic Rossby waves, especially the long ones that range from 1000 to $10,000 \mathrm{~km}$ in length and from months to decades in period, carry energy and information from the atmospheric forcing to remote regions. Together with Kelvin waves - long gravity waves along land-sea boundaries and the equator, Rossby waves tend to set the ocean toward equilibrium with a varying atmosphere. Thus, the study of Rossby waves has become a major foundation in modern dynamical meteorology and oceanography [Pedlosky, 1987].

[3] The transient nature of Rossby waves makes them difficult to be observed from traditional observing instruments that have been deployed, typically along a few lines or at a few stations. The study of oceanic Rossby waves remained largely theoretic with important contributions from numerical models and laboratory experiments until

\footnotetext{
${ }^{1}$ Physical Oceanography Laboratory, Ocean University of China, Qingdao, China.

${ }^{2}$ Department of Physical Oceanography, Woods Hole Oceanographic Institution, Woods Hole, Massachusetts, USA.
}

Copyright 2008 by the American Geophysical Union. 0094-8276/08/2008GL034312\$05.00 the advent of high-accuracy satellite altimeters in the 1990's when changes of SSH as little as $1 \sim 2 \mathrm{~cm}$ became observable. The continuous and basin-wide observations of SSH since 1992 make it possible to test important characteristics derived from the wave theory. One major finding is the revealing of global existence of oceanic Rossby waves [Chelton and Schlax, 1996]. This intriguing discovery has prompted numerous studies and led to major improvements of theoretic models [Killworth et al., 1997; Qiu et al., 1997; Dewar, 1998; de Szoeke and Chelton, 1999; Liu, 1999; LaCasce and Pedlosky, 2004].

[4] Despite a large number of studies in the last 15 years, satellite altimetry data continue to provide invaluable information for studying a transient ocean. New features of the ocean are being discovered and new aspects of the exiting theory are being recognized [Fu and Chelton, 2001]. A research area of increasing interest is the geographic distribution of SSH variances. Statistical analyses revealed a fascinating pattern that the spectrum of the SSH variability tends to peak at the same frequency and at the same latitude regardless of which hemisphere or oceanic basin. This results in a nearly zonally-uniform distribution of peakspectrum frequency. For instance, the power spectrum of SSH peaks at the wave period of about 3 months along both $20^{\circ} \mathrm{N}$ and $20^{\circ} \mathrm{S}$ in the Pacific, Atlantic and Indian Oceans [Liu and Wang, 1999; Polito and Liu, 2003; Qiao et al., 2004]. This 3-month peak in SSH has also been found in the tidal station record [Mitchum, 1995; Zhang et al., 2001; Lin et al., 2004]. In general, the energy spectrum peaks at a longer period (lower frequency) along a higher latitude. A dynamical explanation is needed for this interesting phenomenon. In this study, we will further quantify the distribution of the peak-spectrum frequency of SSH variability by using a longer record of SSH than in previous studies, and more importantly, explain the frequency distribution in terms of oceanic dynamics.

\section{Data and Methods}

[5] For this study, we use the latest version of global SSH anomaly dataset compiled by the CLS Space Oceanographic Division of Toulouse, France. The data are the Maps of Sea Level Anomaly of Archiving, Validation and Interpretation of Satellite Oceanographic Data. The dataset merges the TOPEX/Poseidon, ERS-1/2, and Jason-1 Follow-On alongtrack SSH measurements for the period from October 1992 to June 2006. It has a 7-day temporal resolution and a $1 / 4 \times$ 1/4 spatial resolution [Le Traon et al., 1998; Le Traon et al., 2001]. The wavelength of a Rossby wave, for a given frequency, shortens poleward. The data resolution becomes insufficient in high latitude oceans, so we will restrict our study to the world ocean between $40^{\circ} \mathrm{S}$ and $40^{\circ} \mathrm{N}$. 
[6] The Fast Fourier Transform [Cooley and Tukey, 1965] is used to calculate the power spectrum of SSH variability, and to identify its peaks at every grid point. There are 712 data at each grid point with a 7-day interval, so we have 356 frequency bands at most and the highest frequency is $2 \pi / 14$ days. The power spectrum of oceanic variability is red and thus increases gradually toward a lower frequency. We are mostly interested in the frequency at which the spectrum peaks. This frequency is usually higher than that associated with the semi-annual cycle (6 month period) in a typical mid-latitude ocean. So the peak frequency of the power spectrum will be identified by comparing the power spectrum in every frequency with the total power spectrum integrated for frequency higher than $2 \pi / 165$ days. We integrated the spectrum percentage into 5 main frequency bands: (a) $2 \pi / 15-2 \pi / 45$ days, (b) $2 \pi / 45-2 \pi / 75$ days, (c) $2 \pi / 75-2 \pi / 105$ days, (d) $2 \pi / 105-2 \pi / 135$ days, and (e) $2 \pi / 135-2 \pi / 165$ days. These represent variability in wave periods of 1, 2, 3, 4 and 5 months respectively.

[7] To further investigate the latitudinal dependence of SSH variability, we also apply the 2-dimensional Fast Fourier Transform to SSH data along each latitude and then project the power spectrum into the wavelength or period space.

\section{Analysis and Results}

[8] Figure 1 shows the spectrum percentage between the one integrated over one of the five selected frequency bands ( $2 \pi / 15-2 \pi / 45$ days, $2 \pi / 45-2 \pi / 75$ days, $2 \pi / 75-2 \pi / 105$ days, $2 \pi / 105-2 \pi / 135$ days, and $2 \pi / 135-2 \pi / 165$ days) and the one integrated over the whole high frequency $(>2 \pi /$ 165 days) range. These five frequency bands represent variability in wave periods around 1,2,3,4 and 5 months respectively. A prominent feature in Figure 1 is the most energetic (or peak-spectrum) variability in each of the selected frequency range is distributed along two zonal bands that are roughly symmetric about the equator. The areas where variability with periods of around 1 month dominates are basically centered along $7^{\circ} \mathrm{N}$ and $7^{\circ} \mathrm{S}$. In higher latitudes, lower-frequency variability becomes dominant. For example, the latitudinal bands centered along $14^{\circ}$ on both hemispheres are dominated by SSH variability with periods of around 2 months. The peak-spectrum bands move poleward to $20^{\circ}$ for a 3 -month period, $26^{\circ}$ for a 4-month period, and to $32^{\circ}$ for a 5 -month period. The $\mathrm{SSH}$ variability within each of the five selected frequency bands usually contributes more than $40 \%$ to the total high-frequency power spectrum along its peak latitudes.

[9] Why does the frequency of the most energetic variability depend on the latitudinal location on either hemisphere? This pattern is unlikely to be imposed directly by the atmosphere. The atmospheric variability typically has much larger spatial scales. Thus, a large spatial range of the ocean in each oceanic basin would have been forced by the same atmospheric variability, and the oceanic response would be different from the fine band structures shown in Figure 1. Furthermore, the atmosphere variability has many regional dominant modes, and is quite different in both structure and frequency over each oceanic basin. The fact that the peak-spectrum frequency, (Figure 1), depends mostly on the latitude, regardless of which hemisphere or oceanic basin, strongly indicates that the structure and location of the most energetic sea surface variability in the ocean is likely determined internally by oceanic dynamics.

[10] In the interior ocean, away from the land-sea boundaries and the equator, oceanic variability with periods longer than a few weeks is governed by the dynamics of Rossby waves. For a stratified ocean, one can decompose Rossby waves into a series of vertical modes with the first baroclinic mode being the most dominant one. The frequency and wavelength of a free Rossby wave are inter-dependent through the dispersion relation. There are two types of Rossby waves at a given frequency that is lower than the critical frequency (its value depends largely on latitude). The energy of long waves, i.e., longer than a few tens of kilometers, propagates westward, while the energy of short ones goes eastward. The long and short waves merge at the critical frequency [Longuet-Higgins, 1968] and the wave energy become stagnant. This critical frequency for a zonally-propagating wave is defined as [Pedlosky, 1987, p. 82]

$$
\omega_{c} \approx \frac{\beta c_{1}}{2 f}
$$

where $f=2 \Omega \sin \theta, \beta=2 \Omega \cos \theta / R$ ( $\Omega$ and $R$ are the rotation rate and the radius of the earth respectively, $\theta$ is the latitude along which a Rossby wave propagates) and $c_{1}$ is the speed of a long gravity wave, and determined by the stratification and depth of the water column. The zonal wavelength at this critical frequency is

$$
L_{c}=\frac{2 \pi c_{1}}{f}
$$

Both the critical frequency and its associated wavelength depend on the latitude $\theta$, and the hydrographic and bathymetric conditions that determine $c_{1}$.

[11] The $\omega_{c}$ is also the maximum frequency that a free Rossby wave can exist along a given latitude $\theta$ and in an environment that determines $c_{1}$ in equation (1). One can also define the critical latitude through equation (1):

$$
\theta_{c}=\cot ^{-1}\left(\frac{2 R \omega}{c_{1}}\right)
$$

For a given frequency, free Rossby waves exist only in latitudes lower than the critical latitude and thus $\theta_{c}$ is also called the turning latitude since poleward propagating Rossby waves would have to turn from this latitude back toward the equator.

[12] Does the latitudinal band structure of the peakspectrum frequency distribution, shown in Figure 1, relate to the critical frequency or the critical latitude? To answer this, we use the water density from a comprehensive observational data set (WOA 01) [Conkright et al., 2002] to compute the long gravity wave speed $c_{1}$ [Chelton et al., 1998]. The solid lines in Figure 1 indicate the position of the critical latitude $\theta_{c}$ for a frequency of $2 \pi / 30$ days (Figure 1a), $2 \pi / 60$ days (Figure $1 \mathrm{~b}$ ), $2 \pi / 90$ days (Figure $1 \mathrm{c}$ ), $2 \pi / 120$ days (Figure 1d) and $2 \pi / 150$ days (Figure 1e), derived by the calculated $c_{1}$. These lines of $\theta_{c}$ match well the latitudinal bands of the peak-spectrum frequencies for all five selected 
a
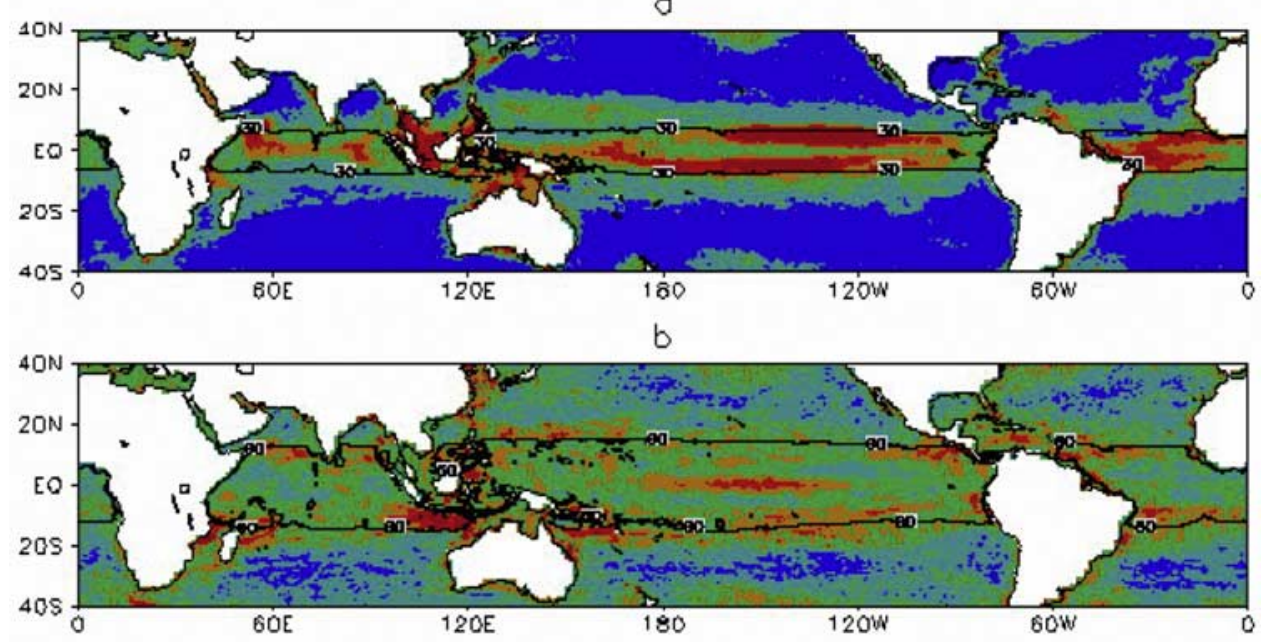

$c$

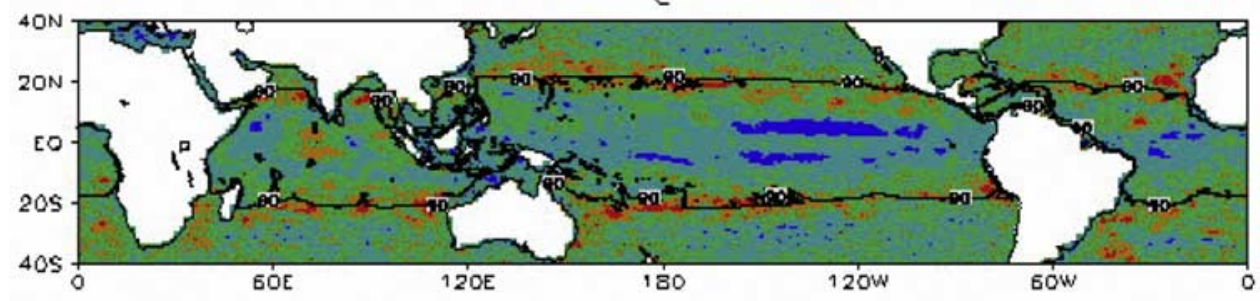

$d$

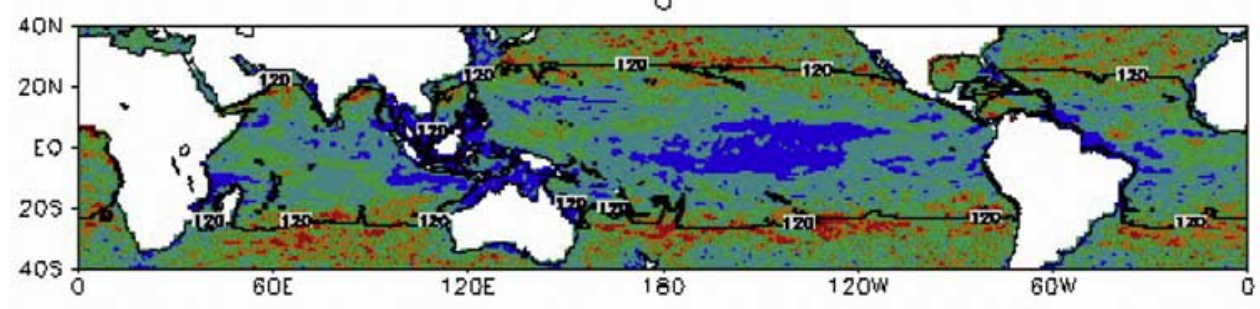

e

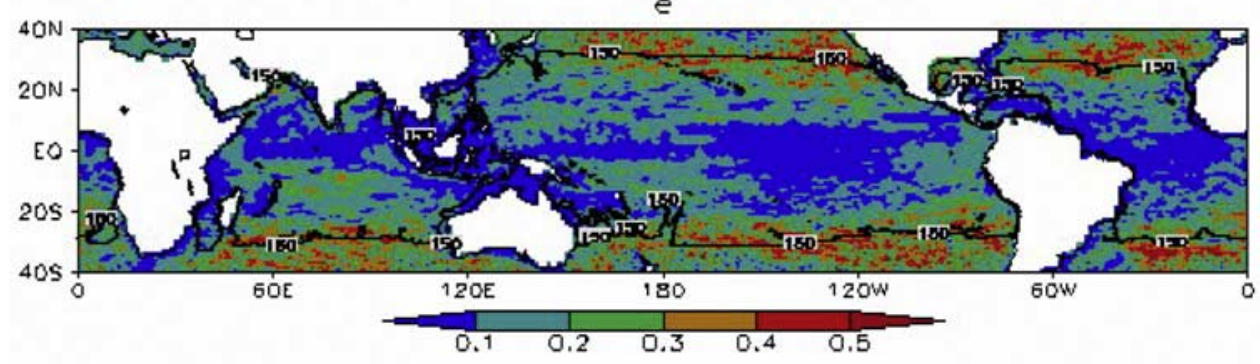

Figure 1. The spectrum percentage (shading) between a selected frequency band and the accumulated one for the frequency higher than $2 \pi / 165$ days. The selected frequency bands are: (a) $2 \pi / 15-2 \pi / 45$ days, (b) $2 \pi / 45-2 \pi / 75$ days, (c) $2 \pi / 75-2 \pi / 105$ days, (d) $2 \pi / 105-2 \pi / 135$ days, and (e) $2 \pi / 135-2 \pi / 165$ days. The data used here are 14-year (19922006) satellite altimetry observation of SSH. Note that there are zonal bands of high-spectrum frequency. The solid lines are the locations of critical latitude $\theta_{c}$ for each of the following frequencies: $2 \pi / 30$ days (Figure 1a), $2 \pi / 60$ days (Figure 1b), $2 \pi /$ 90 days (Figure 1c), $2 \pi / 120$ days (Figure 1d), and $2 \pi / 150$ days (Figure 1e). Along a critical latitude $\theta_{c}$ the energy propagation of a Rossby wave becomes stagnant. The value of $\theta_{c}$ is calculated by using a hydrographic data set (WOA 01) which is independent from the altimetry data. It is obvious that the frequency of the peak-spectrum SSH variability (shaded) is very close to the critical frequency of Rossby waves (solid lines). In this report, we offer a dynamical explanation for this relationship.

frequency ranges. How do we explain this close match between $\theta_{c}$ and the data-derived latitude of peak-spectrum frequency? Is this nearly perfect match merely coincidental or dynamically linked?
[13] The atmosphere changes constantly, and therefore its variability have a wide range of frequency. For those atmospheric variations that have frequencies higher than the critical frequency $\omega_{c}$, the Rossby waves do not exist. For 


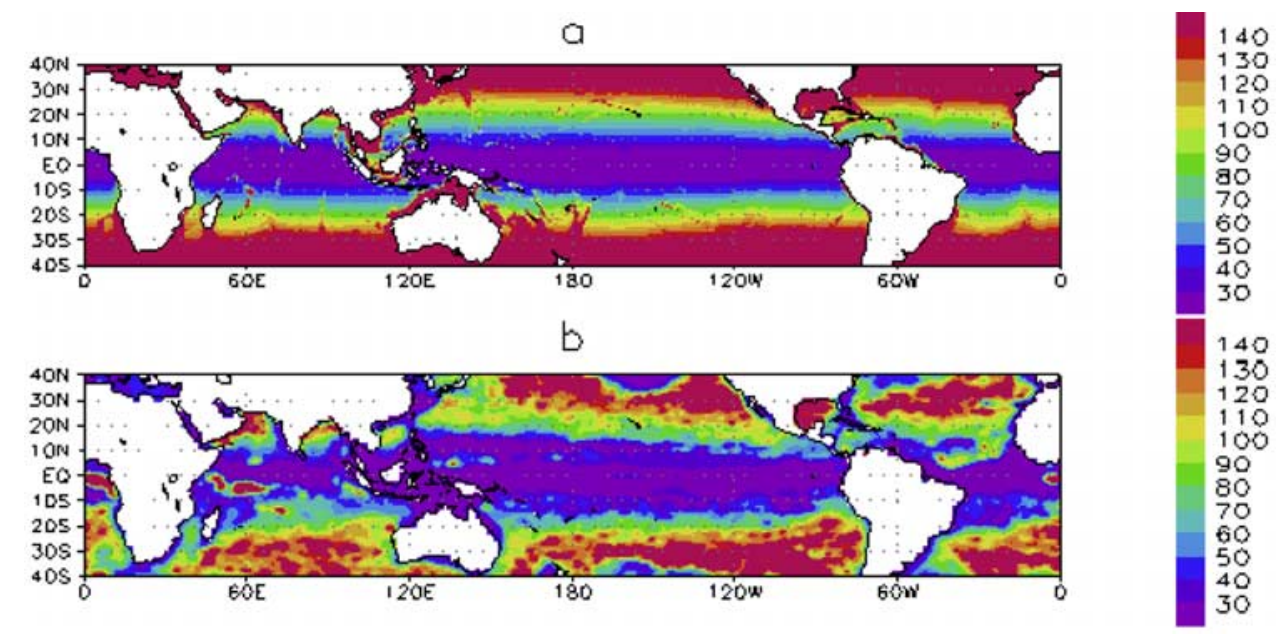

Figure 2. (a) The global distribution of the critical period, $2 \pi / \omega_{\mathrm{c}}$ (days), calculated from WOA 01 data and (b) the period of peak-spectrum variability (days) of altimetry SSH data. There is remarkable similarity, both qualitatively and quantitatively, between the frequency distribution of most energetic (peak-spectrum) SSH variability (Figure 2b) and the critical Rossby-wave frequency (Figure 2a). This good match demonstrates that SSH variability is significantly more energetic at the critical frequency when the zonal energy propagation of Rossby waves becomes stagnant, and thus the energy flux from the atmosphere accumulates locally in the interior ocean.

those with frequencies considerably lower than $\omega_{c}$, however, both long and short oceanic Rossby waves transmit the energy from the atmospheric forcing in the ocean interior toward the western and eastern oceanic boundaries. But for variability with frequency near or at $\omega_{c}$, the oceanic responses are slow or stagnant zonal propagation of energy away from the forcing regions. So the energy from the atmospheric forcing may accumulate locally in the ocean interior. The solid lines in Figures 1a-1e show the locations where the first baroclinic mode Rossby waves become zonal stagnant for the five chosen frequency bands. The good match between them, and the peak-spectrum SSH variability indicates that the SSH variability is significantly more energetic near the critical frequency $\omega_{c}$. We would like to note here that waves with zero zonal group velocity can still propagate in the meridional direction and their speed is roughly proportional to $1 / L_{\theta}$ (where $L_{\theta}$ is the wavelength in the meridional direction). So the peak spectrum along the critical latitude is due mostly to those with long (longer than $100 \mathrm{~km}$ in a mid-latitude ocean) wavelengths in the meridional direction. Waves with shorter meridional wavelengths turn equatorward from the critical latitude and may become zonally-propagating waves at lower latitudes. They do not affect directly the existence of those with longer meridional wavelengths that lead to the observed spectrum peaks.

[14] The relationship between the peak-spectrum frequency and the critical Rossby wave frequency can further be illustrated in Figure 2. The distribution of $2 \pi / \omega_{c}$ (Figure 2a), the wave period associated with the critical frequency computed from WOA 01 data set, shows that the critical frequency (period) is rather uniform zonally and decreases (increases) from the equator to the poles. The SSH altimetry data, from 1992 to 2006, was used to determine where the peak-spectrum variability is located for a given frequency from $2 \pi / 30$ days to $2 \pi / 150$ days. The geographic distribution of the wave period at which the spectrum peaks, Figure $2 \mathrm{~b}$, is very similar, both qualitatively and quantitatively, to that of $2 \pi / \omega_{c}$ shown in Figure 2a. We would like to note that there are some discrepancies, mostly in the high latitude, between the two estimates. But there is overall good agreement between Figures $2 \mathrm{a}$ and $2 \mathrm{~b}$ which supports our hypothesis that the peak-spectrum variability of the satelliteobserved SSH is associated with the zonal stagnant Rossby waves at their critical frequencies.

[15] The global SSH data provides not only the temporal variability, but also the spatial one. So one can also calculate the peak-spectrum wavelengths and test whether they are consistent with critical wavelengths expected from wave theory. Figures $3 \mathrm{a}$ and $3 \mathrm{~b}$ are the power spectrum in wavelength-latitude and period-latitude space from $\mathrm{SSH}$ data. The solid lines in Figure 3 are the corresponding critical wavelength, defined by equation (2), and the corresponding critical period calculated by using $c_{1}$ derived from WOA 01 data. The high-spectrum band of the $\mathrm{SSH}$ variability is basically centered along the critical wavelength $L_{c}$ in Figure $3 \mathrm{a}$ or along the critical wave period $2 \pi / \omega_{c}$ in Figure $3 \mathrm{~b}$. So the temporal and spatial structures of the peak-spectrum SSH variability are indeed related through the theoretically-expected dispersion relation of Rossby wave.

\section{Summary and Discussion}

[16] A recent study [Chelton et al., 2007] shows that more than $50 \%$ of the variability over much of the World Ocean is accounted for by eddies with amplitudes of 5$25 \mathrm{~cm}$ and diameters of $100-200 \mathrm{~km}$. The phases of these eddies propagate mainly westward. They were interpreted as nonlinear Rossby waves because their small excursions in the meridional directions are consistent with characteristics of nonlinear vortices [McWilliams and Flierl, 1979] even though it is difficult to diagnose their nonlinearity from the SSH data alone. The half wavelength of a stagnant Rossby wave (or the diameter of an eddy-like feature), as defined by equation (2), would be within the $100-200 \mathrm{~km}$ range poleward of 20 degree in latitude (Figure 3a). Here 
a

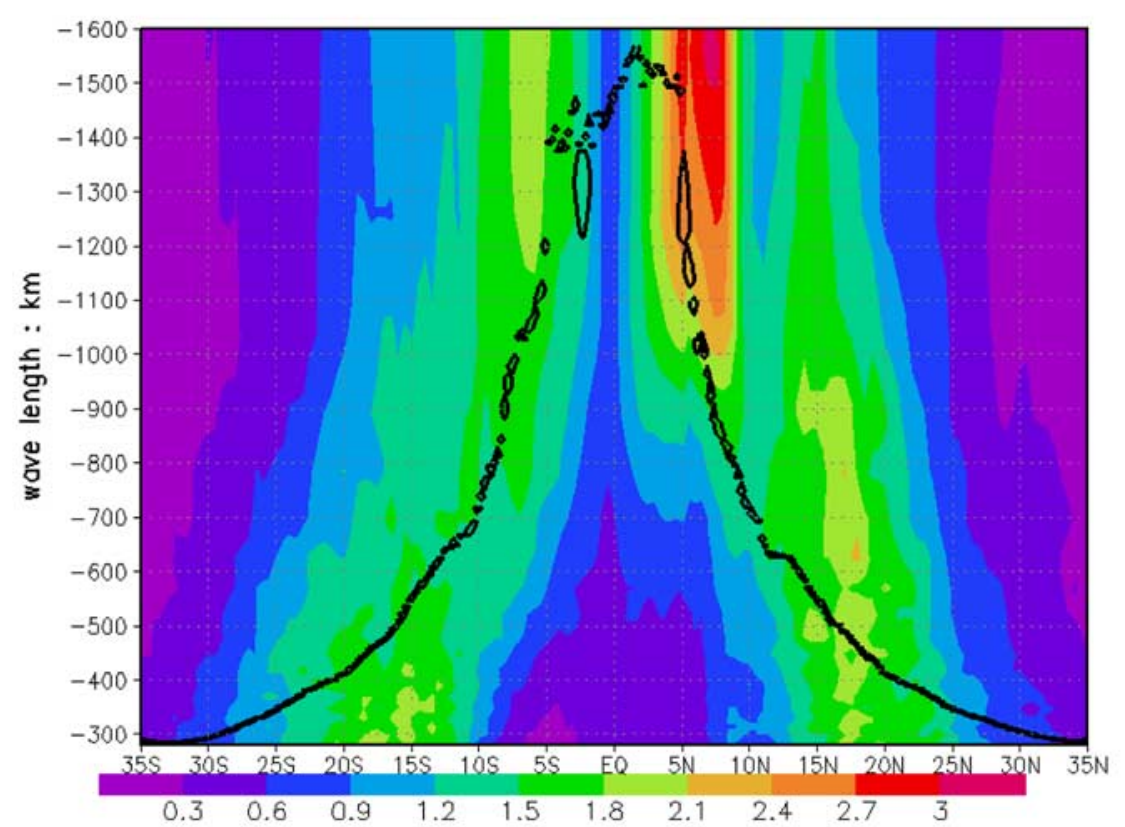

b

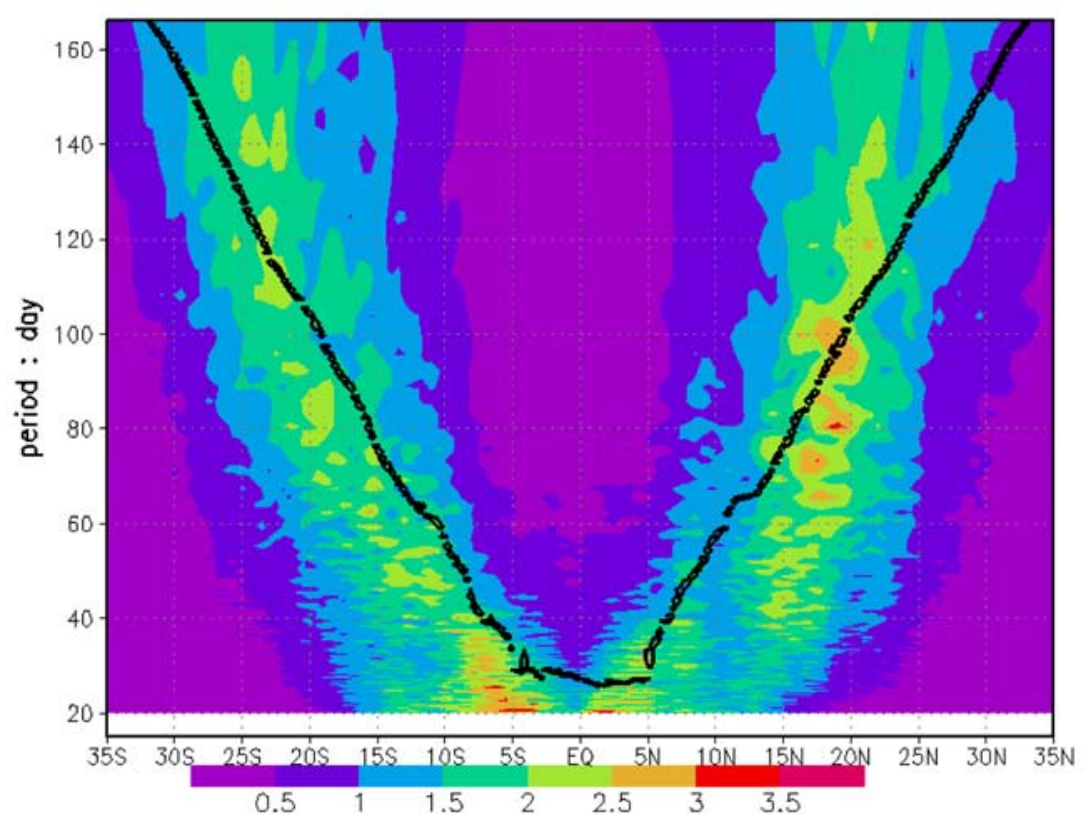

Figure 3. (a) The wavelength-latitude power spectrum and (b) period-latitude power spectrum from SSH data (shaded). The solid lines are the corresponding critical wave length, defined by equation (2), and the corresponding critical period from WOA 01 data.

we offer an alternative explanation for the existence of those high-energy variations. We must also point out here that the phase of an energy-stagnant Rossby wave still travels westward. This aspect does not contradict the previous finding. Furthermore, satellite altimetry data only resolve low-frequency waves and the inertial gravity waves are excluded in our analyses. One may expect the existence of similar spectrum peaks associated with high-frequency inertial gravity waves. In fact, Wunsch and Gill [1976] attributed spectrum peaks in the observed SSH in tidal stations in the tropical ocean to stagnant inertial waves with zero group velocity.

[17] Acknowledgments. This work was mainly conducted during a visit by X.L. to Woods Hole Oceanographic Institution as a guest investigator. We have benefited from talking with Joe Pedlosky, Terry Joyce, and John Toole. We also thank Dr. Shang-ping Xie, Dr. Bo qui at the University of Hawaii, Dr. Zhengyu Liu at the University of Wisconsin-Madison and two anonymous reviewers for their helpful comments. This study is 
supported by China's National Basic Research Priorities Programmer (2005CB422303 and 2007CB411804), the key project of the International Science and Technology Cooperation program of China (2006DFB21250), the Ministry of Education's 111 Project (B07036), the Program for New Century Excellent Talents in University (NECT-07-0781), and the US National Science Foundation (OCE-0351055).

\section{References}

Anderson, D. L. T., and A. E. Gill (1975), Spin-up of a stratified ocean, with application to upwelling, Deep Sea Res., 22, 583-596.

Chelton, D. B., and M. G. Schlax (1996), Global observations of oceanic Rossby waves, Science, 272, 234-238.

Chelton, D. B., R. A. deSzoeke, M. G. Schlax, K. El Naggar, and N. Siwertz (1998), Geographical variability of the first baroclinic Rossby radius of deformation, J. Phys. Oceanogr., 28, 433-460.

Chelton, D. B., M. G. Schlax, R. M. Samelson, and R. A. de Szoeke (2007), Global observations of large oceanic eddies, Geophys. Res. Lett., 34, L15606, doi:10.1029/2007GL030812.

Conkright, M. E., R. A. Locarnini, H. E. Garcia, T. D. O’Brien, T. P. Boyer, C. Stephens, and J. I. Antonov (2002), World Ocean Atlas 2001: Objective Analyses, Data Statistics, and Figures, CD-ROM Documentation, 17 pp., NOAA, Silver Spring, Md.

Cooley, J. W., and J. W. Tukey (1965), An algorithm for the machine calculation of complex Fourier series, Math. Comput., 19, 297-301.

de Szoeke, R. A., and D. B. Chelton (1999), The modification of long planetary waves by homogeneous potential layers, J. Phys. Oceanogr., $29,500-511$.

Dewar, W. K. (1998), On "too fast" baroclinic planetary waves in the general circulation, J. Phys. Oceanogr., 28, 1739-1758.

Fu, L. L., and D. B. Chelton (2001), Large-scale ocean circulation, in Satellite Altimetry and Earth Sciences: A Handbook for Techniques and Applications, Int. Geophys. Ser, vol. 69, edited by L.-L. Fu and A. Cazenave, pp. 133-169, Academic, San Diego, Calif.

Killworth, P. D., D. B. Chelton, and R. A. de Szoeke (1997), The speed of observed and theoretical long extratropical planetary waves, J. Phys. Oceanogr., 27, 1946-1966.

LaCasce, J. H., and J. Pedlosky (2004), The instability of Rossby basin modes and the oceanic eddy field, J. Phys. Oceanogr., 34, 2027-2041.

Le Traon, P. Y., F. Nadal, and N. Ducet (1998), An improved mapping method of multisatellite altimeter data, J. Atmos. Oceanic Technol., 15, $522-534$.
Le Traon, P.-Y., G. Dibarboure, and N. Ducet (2001), Use of a high-resolution model to analyze the mapping capabilities of multiple-altimeter missions, J. Atmos. Oceanic Technol., 18, 1277-1288.

Lin, X. P., D. W. Wu, and J. Lan (2004), The intrusion and influence of intraseasonal long Rossby waves in the East China Sea, J. Hydrodyn., Ser. $B, 16,621-631$.

Liu, Q. Y., and Q. Wang (1999), Spatial distribution of the sea surface height intraseasonal oscillation in the tropical Pacific, J. Ocean Univ. Qingdao, 29(4), 549-555.

Liu, Z. (1999), Forced planetary wave response in a thermocline gyre, J. Phys. Oceanogr., 29, 1036-1055.

Longuet-Higgins, M. S. (1964), Planetary waves on a rotating sphere, Philos. Trans. R. Soc. London, Ser. A, 279, 446-473.

Longuet-Higgins, M. S. (1965), Planetary waves on a rotating sphere II, Philos. Trans. R. Soc. London Ser. A, 284, 40-68.

Longuet-Higgins, M. S. (1968), The eigenfunctions of Laplace's tidal equations over a sphere, Philos. Trans. R. Soc. London, Ser. A, 262, 511-607.

McWilliams, J. C., and G. R. Flierl (1979), On the evolution of isolated, nonlinear vortices, J. Phys. Oceanogr., 9, 1155-1182.

Mitchum, G. T. (1995), The source of 90-day oscillations at Wake Island, J. Geophys. Res., 100(C2), 2459-2475.

Pedlosky, J. (1987), Geophysical Fluid Dynamics, 710 pp., Springer, New York.

Polito, P. S., and W. T. Liu (2003), Global characterization of Rossby waves at several spectral bands, J. Geophys. Res., 108(C1), 3018, doi:10.1029/ 2000JC000607.

Qiao, F. L., E. Tal, and Y. L. Yuan (2004), Zonal distribution features of high frequency planetary waves in the oceans derived from satellite altimeter data, Acta Oceanol. Sin., 23(1), 91-96.

Qiu, B., W. Miao, and P. Muller (1997), Propagation and decay of forced and free baroclinic Rossby waves in off-equatorial oceans, J. Phys. Oceanogr., 27, 2405-2417.

Wunsch, C., and A. E. Gill (1976), Observation of equatorially trapped waves in Pacific sea level variations, Deep Sea Res., 23, 371-390.

Zhang, D. X., T. N. Lee, W. E. Johns, C. T. Liu, and R. Zantopp (2001), The Kuroshio east of Taiwan: Modes of variability and relationship to interior mesoscale eddies, J. Phys. Oceanogr., 31, 1054-1074.

X. Lin, D. Wu, and P. Zhai, Physical Oceanography Laboratory, Ocean University of China, Qingdao, 266003, China. (linxiaop@ouc.edu.cn)

J. Yang, Department of Physical Oceanography, Woods Hole Oceanographic Institution, Woods Hole, MA 02543, USA. 